\title{
GA-A23087 \\ CONF-990411-- \\ POLARIZATION, PROPAGATION, AND DEPOSITION MEASUREMENTS DURING ECCD EXPERIMENTS ON THE DIII-D TOKAMAK
}

\author{
by \\ C.C. PETTY, T.C. LUCE, M.E. AUSTIN, \\ Y.-R. LIN-LIU, J. LOHR, R. PRATER
}

DISTRIBUTON OF THIS DOCUMENT is UMMUTED $h$ MASTER

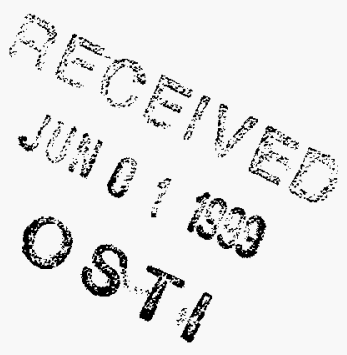

MARCH 1999 


\section{DISCLAIMER}

This report was prepared as an account of work sponsored by an agency of the United States Government. Neither the United States Government nor any agency thereof, nor any of their employees, make any warranty, express or implied, or assumes any legal liability or responsibility for the accuracy, completeness, or usefulness of any information, apparatus, product, or process disclosed, or represents that its use would not infringe privately owned rights. Reference herein to any specific commercial product, process, or service by trade name, trademark, manufacturer, or otherwise does not necessarily constitute or imply its endorsement, recommendation, or favoring by the United States Government or any agency thereof. The views and opinions of authors expressed herein do not necessarily state or reflect those of the United States Government or any agency thereof. 


\section{DISCLAIMER}

Portions of this document may be illegible in electronic image products. Images are produced from the best available original document. 


\title{
POLARIZATION, PROPAGATION, AND DEPOSITION MEASUREMENTS DURING ECCD EXPERIMENTS ON THE DIII-D TOKAMAK
}

\author{
by \\ C.C. PETTY, T.C. LUCE, M.E. AUSTIN, ${ }^{*}$ \\ Y.-R. LIN-LIU, J. LOHR, R. PRATER
}

This is a preprint of a paper to be presented at the 13th Topical Conference on Applications of RF Power to Plasmas, April 12-14, 1999, Annapolis, Maryland and to be published in the Proceedings.

*University of Texas, Austin, Texas.

\author{
Work supported by \\ the U.S. Department of Energy \\ under Contract No. DE-AC03-99ER54463
}

GA PROJECT 30033

MARCH 1999 


\begin{abstract}
The power deposition profiles for different poloidal and toroidal launch angles have been determined by modulating the ECH power and measuring the electron temperature response. The peak of the measured power density follows the poloidal steering of the ECH launcher, and perpendicular launch gives a narrower deposition profile than does oblique (current drive) launch. The difference in wave refraction between $\mathrm{X}$-mode and $\mathrm{O}$-mode allows positive identification of an unwanted $\mathrm{O}$-mode component of the launched beam.
\end{abstract}

\title{
INTRODUCTION
}

Electron cyclotron heating (ECH) and current drive (ECCD) experiments on DIII-D for advanced tokamak scenarios need narrow beams in nearly pure X-mode to satisfy the requirement of highly localized deposition and current drive. To date, these ECCD experiments have utilized two $110 \mathrm{GHz}$ gyrotrons (corresponding to the second harmonic of the electron cyclotron frequency) that are capable of $1.7 \mathrm{MW}$ of combined power for $1 \mathrm{~s}$ pulses. The electron cyclotron waves are launched from the low-field-side of the torus with a toroidal angular component of $19^{\circ}$ for current drive; perpendicular launch is also possible using a different mirror geometry. The ECCD launcher is steerable in poloidal angle which allows the deposition location to be varied from the plasma center to the edge without altering the plasma parameters such as toroidal magnetic field strength. The polarization of the launched wave required for coupling to the $\mathrm{X}$-mode is controlled by adjusting the inclination of grooved mirrors located in two consecutive mitre bends of the evacuated waveguide (1).

Initial ECH experiments on DIII-D have measured the polarization, propagation, and deposition of the electron cyclotron waves. The power deposition profile is determined experimentally by modulating the gyrotron power at $\sim 100 \mathrm{~Hz}$ and measuring the electron temperature $\left(T_{e}\right)$ response using a 32-channel heterodyne radiometer. In the limit of infinite modulation frequency $(\omega)$, the temperature response $(\widetilde{T})$ is everywhere proportional to the ECH source term with a $90^{\circ}$ phase lag from the injected power. However, in practice the dynamic range of the radiometer is reached before $\omega$ is high enough to make transport effects negligible; experiments on DIII-D find that the apparent power deposition profile narrows continuously with increasing ECH modulation frequency from $50-300 \mathrm{~Hz}$. For square wave modulation, the power deposition profile can be found from (2)

$$
P_{\mathrm{abs}}=\frac{2}{\pi} \omega \frac{3}{2} n_{e} \tilde{T}_{e},
$$

but this places only an upper bound on the deposition profile width due to the relatively low modulation frequency.

\section{EXPERIMENTAL RESULTS}

The ECH deposition location has been verified as the poloidal angle of the launcher is varied using modulation techniques. A toroidal magnetic field strength of $1.9 \mathrm{~T}$ is chosen to optimize the radial coverage of the radiometer, which places the second harmonic resonance near the plasma axis. Figure 1(a) shows the vacuum ray paths, projected onto the poloidal plane, for various poloidal steerings of the launcher (controlled by changing the thickness of a shim). In the toroidal direction, the waves are launched at a fixed oblique angle for current drive, thus the waves can make two passes through the plasma before striking a solid surface. The measured ECH power density from 

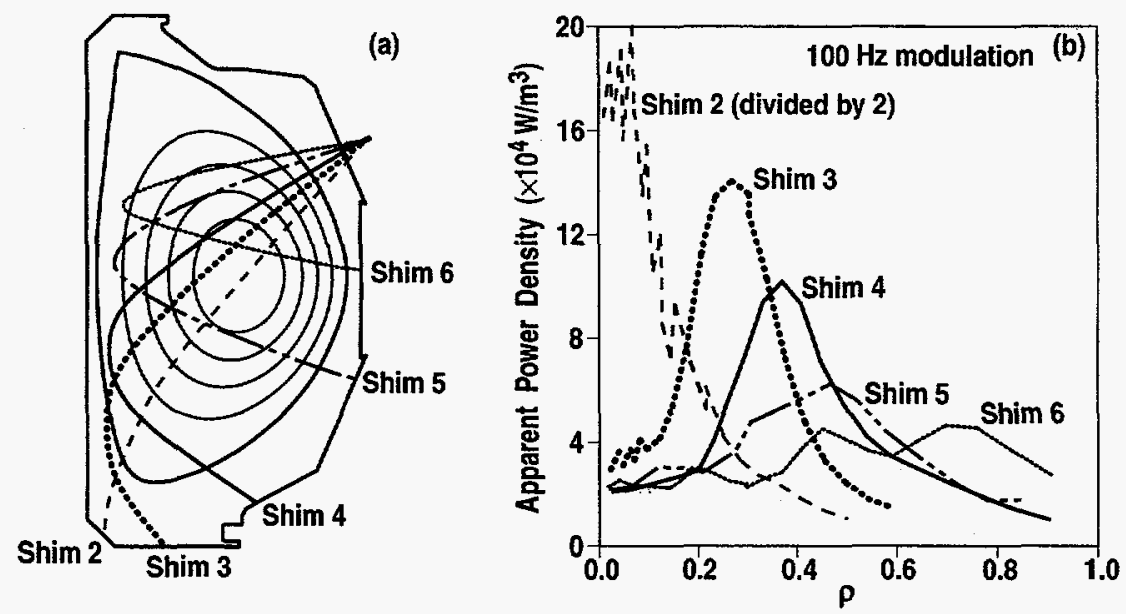

FIGURE 1. (a) Vacuum ray paths and (b) experimental power deposition profiles for a scan of the poloidal steering of the ECCD launcher.

Eq. (1) for a modulation frequency of $100 \mathrm{~Hz}$ is shown as a function of the normalized radius $(\rho)$ in Fig. 1(b). The maximum power density is seen to follow the beam steering. However, a secondary peak in the power deposition profile is also sometimes observed which may be due to imperfect polarization of the launched waves.

Determination of the polarization of the electron cyclotron waves is possible since the wave refraction for $\mathrm{X}$-mode and $\mathrm{O}$-mode is noticeably different even in low density plasmas; therefore, the $\mathrm{X}$-mode and $\mathrm{O}$-mode powers are deposited in different regions of the plasma. Figure 2 (a) shows the ray paths for $\mathrm{X}$-mode and $\mathrm{O}$-mode beams with off-axis steering, both of which are significantly different from the vacuum ray path. The power density profile measured for this case (with nominal X-mode polarization) is shown in Fig. 2(b) along with the theoretical deposition profiles for
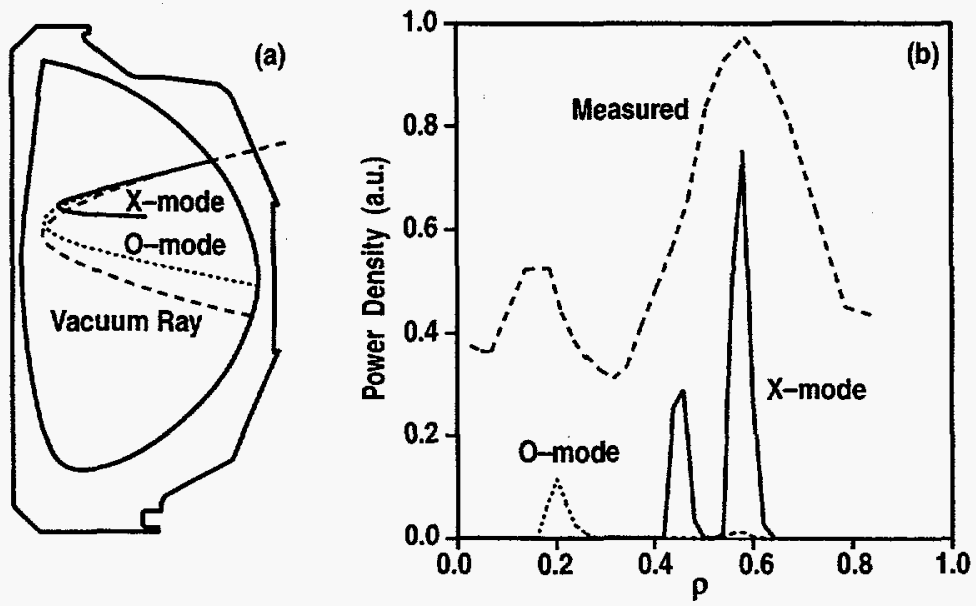

FIGURE 2. (a) Ray paths for vacuum, X-mode, and $\mathrm{O}$-mode, and (b) experimental power deposition profile. The theoretical deposition locations for $\mathrm{X}$-mode and $\mathrm{O}$-mode along the ray paths are also shown. 
$\mathrm{X}$-mode and $\mathrm{O}$-mode calculated using the TORAY ray tracing code (3). Both polarizations make two passes through the plasma due to the oblique toroidal launch angle, but while the $\mathrm{X}$-mode power is always strongly absorbed, the $\mathrm{O}$-mode power is weakly absorbed on the first pass owing to the low electron temperature; the $\mathrm{O}$-mode power is better absorbed on the second pass since it travels closer to the plasma center. The experimental power density profile in Fig. 2(a) shows clear evidence of an $\mathrm{O}$-mode component in the launched waves. This $\mathrm{O}$-mode component is most likely due to imperfect polarization of the launched beam rather than mode conversion along the ray path.

In experiments using two different toroidal angles for the ECH launcher, the absorption profile for perpendicular launch is measured to be significantly narrower than for oblique launch. Although current drive experiments require oblique launch of the waves, many transport experiments prefer perpendicular launch owing to the more localized heating profile. Figure 3 shows the measured power deposition profiles for both perpendicular and oblique launch; the $\mathrm{ECH}$ power modulation frequency is the same $(100 \mathrm{~Hz})$ for both cases. The theoretical absorption profiles from the TORAY ray tracing code are also shown for comparison. The deposition profiles for both perpendicular and oblique launch show complete absorption of the electron cyclotron waves and good accountability of the injected power, but the oblique launch case has much wider wings to the heating profile which leads to a decrease in the peak power density.

One complication in interpreting the power deposition profiles determined from these modulation experiments is that vertical motion of the plasma can mimic a heat source, especially in regions where the heat pulse amplitude is small $\left(T_{e}<10 \mathrm{eV}\right)$. In Figs. 1-3, it is seen that the apparent power deposition profile never goes to zero, even in regions far from the $\mathrm{ECH}$ resonance. Modulation experiments at reduced magnetic field with the ECH resonance on the high-field-side of the axis still detect a $T_{e}$ response in the plasma center that is in phase with the off-axis deposition, as shown in Fig. 4. This cannot be due to the wrong polarization since there is no resonance near the axis in this situation. In addition, this apparent heat source far from the resonance cannot be due to radial motion of the plasma because points on both the high-field-side and lowfield-side of the axis see this effect in phase. The most likely explanation of this effect is vertical motion of the plasma in synch with the ECH modulation, which gives rise to an apparent $T_{e}$ due to the vertical gradient in $T_{e}$ along the viewing sight of the radiometer. This effect must be taken into account to interpret the power deposition profiles.

\section{CONCLUSIONS}

The measured propagation and deposition of electron cyclotron waves on the DIII-D tokamak reported here and elsewhere (4) are in agreement with theoretical modeling. Scans of the poloidal steering of the ECH launcher (as well as the toroidal magnetic field strength) have been used to calibrate the ray paths for ECCD experiments. The narrower deposition profile for perpendicular launch compared to oblique (current drive) launch means that the former is preferred for heating and transport experiments. Further work is necessary to eliminate the unwanted $\mathrm{O}$-mode component of the beam; some success has been obtained by scanning the polarizer settings to minimize the $\mathrm{O}$-mode peak in the measured power deposition profile.

This work was supported by the U.S. Department of Energy under Contract No. DE-AC03-99ER54463. 


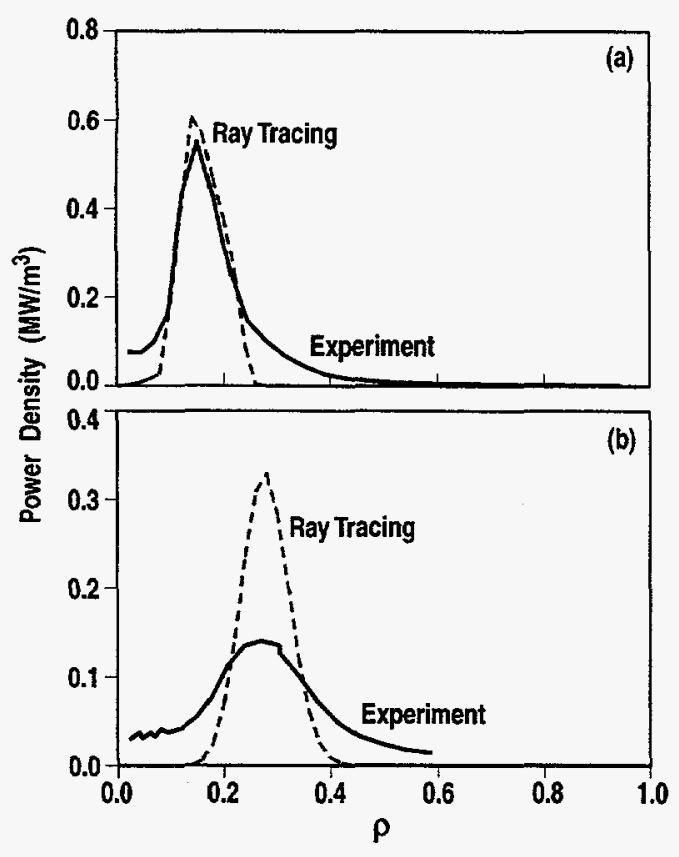

FIGURE 3. Experimental power deposition profiles for (a) perpendicular launch, and (b) oblique launch. The theoretical deposition profiles are shown for comparison.

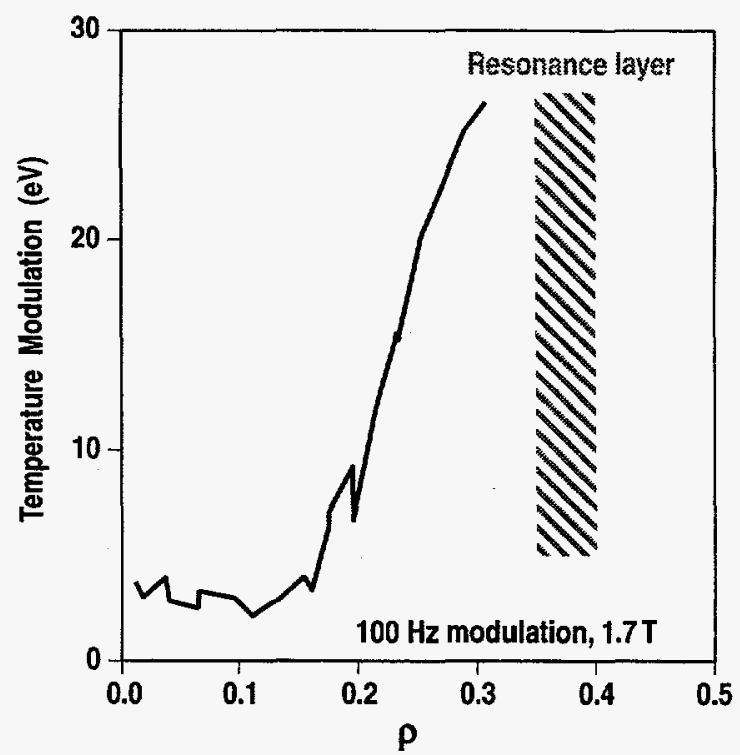

FIGURE 4. Modulated electron temperature as a function of normalized radius when the ECH resonance is located on the high-field-side of the axis.

\section{REFERENCES}

1. Doane, J., Int. J. Infrared Millimeter Waves 13, 1727 (1992).

2. Murakami, M., et al., Proc. 10th Int. Conf. on Radio Frequency Power in Plasmas, Boston (AIP, 1993), p. 48.

3. Matsuda, K., IEEE Trans. Plasma Sci. PS-17, 6 (1989).

4. Prater, R., et al., Proc. 25th Euro. Conf. on Controlled Fusion and Plasma Physics, Praha (EPS, 1998), Vol. 22C, p. 1410. 